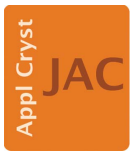

JOURNAL OF

APPLIED

CRYSTALLOGRAPHY

ISSN 1600-5767

\title{
Wavelength-dispersive dark-field contrast: micrometre structure resolution in neutron imaging with gratings
}

\author{
M. Strobl, ${ }^{\text {a,b* }}{ }^{*}$ B. Betz, ${ }^{\text {c }}$ R. P. Harti, ${ }^{c}$ A. Hilger, ${ }^{d}$ N. Kardjilov, ${ }^{\text {d }}$ I. Manke ${ }^{d}$ and \\ C. Gruenzweig ${ }^{\mathrm{c}}$
}

Received 18 December 2015

Accepted 18 February 2016

a'European Spallation Source AB, Lund, 22100, Sweden, ${ }^{\mathbf{b}}$ The Niels Bohr Institute, University of Copenhagen,

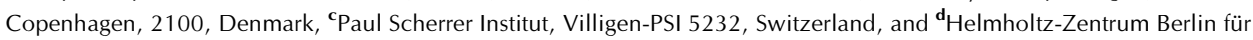
Materialien und Energie GmbH, Berlin, 14109, Germany. ${ }^{*}$ Correspondence e-mail: markus.strobl@esss.se

Edited by D. I. Svergun, European Molecular Biology Laboratory, Hamburg, Germany

Keywords: neutron imaging; dark-field imaging; grating interferometry; small-angle scattering; wavelength-dispersive neutron imaging.

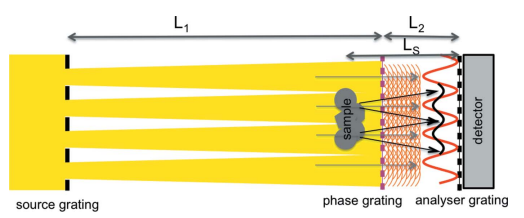

(C) 2016 International Union of Crystallography
Dark-field imaging using grating interferometers has been proven to have a high potential for applications in engineering, magnetism, and soft matter and biophysics, as well as in medicine with both neutrons and X-rays. The access to spatially resolved small-angle scattering information in neutron dark-field imaging provides information about structures beyond direct spatial image resolution. The dark-field contrast modality is hence a valuable tool for materials science based on neutron imaging. This is underlined by the success of the method, despite its current limitation to qualitative scattering information. Here it is demonstrated how a wavelength-dispersive approach allows such drawbacks to be overcome by providing quantitative structure size information and hence can introduce novel possibilities and insights for materials science.

\section{Introduction}

The basic principle of neutron dark-field imaging (Strobl et al., 2008) with grating interferometers (Pfeiffer et al., 2006) is that small angular beam deviations can be resolved by spatially modulating the neutron beam using gratings. Hence, smallangle scattering (SAS), which involves redistributing intensity between the bright and dark areas of the modulated beam, can be detected as a loss of modulation visibility (Fig. 1). The detectable scattering signal in the 'dark field' of the modulation lends its name to the method. In particular, when the spatial beam modulation is of a smaller period than the spatial resolution of an imaging setup, such an approach can be exploited in neutron imaging in a straightforward manner with a conventional pinhole imaging geometry. This enables much higher efficiency (Strobl et al., 2008; Pfeiffer et al., 2006) than earlier approaches utilizing mainly perfect crystal neutron optics to detect SAS in neutron imaging (Strobl, Treimer \& Hilger, 2004). In contrast to the grating interferometer, the crystal optics required very high coherence conditions and hence an unfavourable utilization of the limited available phase space density of neutron sources. Consequently the grating setup constituted major progress. A neutron Talbot Lau interferometer consists of a set of three gratings (Fig. 1). A first absorption grating, the source grating, installed close to the pinhole of the imaging instrument, provides partial coherence sufficient to achieve a distinct interference pattern induced by a phase grating further downstream, close to the detector position. The periods and distances are chosen such that the interference patterns of the individual and partially coherent beams from the source grating superpose constructively at the position of the analyser grating, at a fractional 
Talbot distance from the phase grating right in front of the imaging detector. The analyser grating matches the period of the modulation of the generated interference pattern and enables resolution of the modulation through a spatial phase stepping scan (Pfeiffer et al., 2006), in particular when the spatial resolution of the detector is not sufficient (Fig. 1). The interference pattern recorded this way in every detector pixel of an imaging detector is a cosine spatial intensity modulation that can locally be characterized by the equation

$$
I(x)=I_{0} \exp (-\mu t)[1+V \cos (2 \pi x / p+\varphi)] .
$$

Here $I_{0}$ is the incident intensity, $x$ the location across a pixel (scanning parameter) and $p=p_{1} / 2$ is the modulation period given by the period $p_{1}$ of a $\pi$ shifting phase grating. The parameters with a dependence on the sample in the beam are $\mu, t, \varphi$ and $V$, the linear attenuation coefficient, the thickness of the sample, the phase shift and the visibility $V=\left[I(x)_{\max }-\right.$ $\left.I(x)_{\min }\right] /\left[I(x)_{\max }+I(x)_{\min }\right]$, respectively. $I(x)_{\max }$ and $I(x)_{\min }$ denote the average maximum and minimum intensities of the modulation in a pixel.

Correspondingly, grating interferometry allows for extracting three independent contrast signals with the spatial resolution of the imaging instrument: namely, (i) conventional attenuation contrast, which is measured by the average intensity of the modulation and hence is characterized by the factor $\exp (-\mu t)$, (ii) differential phase contrast (Pfeiffer et al., 2006), as the parameter $\varphi$ describes local phase shifts induced by refraction of the beam in the sample (Strobl, Treimer, Hilger \& Feye-Treimer, 2004), and finally (iii) dark-field contrast (Strobl et al., 2008) with respect to the visibility contrast $V$, which relates to small-angle scattering (Feigin \& Svergun, 1987) effects due to the interaction of the neutrons with microscopic sample structures beyond the real space resolution power of neutron imaging.

Hence the pixel-wise extraction of the parameter $V$, referred to as dark-field imaging, is a measure of the local small-angle scattering power. This technique has been applied successfully in neutron imaging in the past. It has to date enabled outstanding insights concerning, for example, inhomogeneities of the microstructure in engineering materials (Hilger et al., 2010), as well as the visualization of magnetic domains in bulk samples in two (Grünzweig et al., 2008) and three dimensions (Manke et al., 2010), and with respect to magnetic structures in the intermediate phase of superconductors (Reimann et al., 2015). This has been achieved

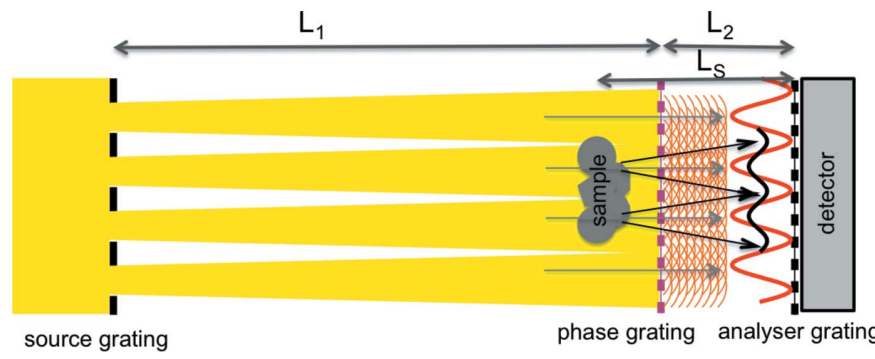

Figure 1

Schematic neutron grating interferometer setup for dark-field imaging through the unique ability to combine qualitative scattering information with real space image resolution. The theory for extracting quantitative SAS information from such neutron dark-field imaging has been provided recently (Strobl, 2014). This adds significant potential to dark-field imaging of inhomogeneous sample systems as it enables small-angle neutron scattering (SANS) characterizations with simultaneous image resolution. Thus, it will provide multi-scale information through SANS in a reciprocal space range of around $5 \times$ $10^{-3} \mathrm{~nm}^{-1}$ combined with real space image resolution of the order of $50 \mu \mathrm{m}$.

\section{Theory}

It has recently been deduced (Strobl, 2014) that the relative local visibility $V_{\mathrm{s}} / V_{0}$, with $V_{0}$ being the intrinsic visibility achieved with a setup and $V_{\mathrm{s}}$ the same quantity with a sample in place, can be written as

$$
V_{\mathrm{s}}\left(\xi_{\mathrm{GI}}\right) / V_{0}\left(\xi_{\mathrm{GI}}\right)=\exp \left\{\int_{\text {path }} \Sigma\left[G\left(\xi_{\mathrm{GI}}\right)-1\right] \mathrm{d} t\right\} .
$$

This contains, besides the total scattering cross section $\Sigma$, a real space correlation function $G$ of the probed microscopic structures. This real space correlation function, which is equivalent to the so-called spin-echo SANS (SESANS) correlation function (Rekveldt, 1996; Andersson et al., 2008), is measured as a function of the instrument-dependent parameter $\xi$. $\xi$ can be referred to as the autocorrelation length or dark-field length (in analogy to the spin-echo length; Rekveldt, 1996) probed in a specific measurement. As this dark-field length

$$
\xi_{\mathrm{GI}}=\lambda L_{\mathrm{s}} / p
$$

depends on the wavelength $\lambda$, the period $p$ and the sample-toanalyser distance $L_{\mathrm{S}}$, it can be used to scan and reveal the real space correlation function $G$ of the probed microscopic sample structures along $\mathbf{x}$, the direction of modulation.

However, the period in the phase grating interferometer setup is fixed. It is coupled to the condition $p_{0}=p L_{1} / L_{2}$ for the source grating period $p_{0}$. This allows constructive superposition to be induced at the analyser grating. In the presented case for a phase shift of $\pi$ with a period $p_{1}$ the modulation period is $p=p_{1} / 2$ and is measured at a fractional Talbot distance $L_{2}=1.94 \mathrm{~cm}$ (Pfeiffer et al., 2006). The latter parameters imply a single wavelength $\lambda$ for which the grating setup is optimized, as the phase shift in the phase grating material is strongly wavelength dependent and so is the Talbot distance. A scan of the sample distance is very limited owing to the short first fractional Talbot distance $L_{2}$ used in current setups, and the spatial resolution is severely deteriorated when a scan is performed between the source and analyser grating. In the latter case equation (3) has to be adapted by replacing $L_{\mathrm{s}}$ with $L_{\mathrm{s}}^{\prime}=\left(L_{1}+L_{2}-L_{\mathrm{s}}\right) L_{2} / L_{1}(\mathrm{Strobl}, 2014)$. For the coverage of a significant correlation length range it is necessary to use large sample-to-detector distances $d$, which leads to a decrease of the image resolution corresponding to an induced blur of $b=$ $(L / D)^{-1} d$. Here $L / D$ is the collimation ratio of the imaging 
setup (Strobl et al., 2009). On the other hand it has been found previously that grating setups are relatively robust in producing useful modulations for a significant range of wavelengths, and hence a mixed approach of wavelength and distance scans has been applied to probe a sufficient range for the presented study. A wavelength-dispersive approach also has to be considered most promising for future efficient exploitation of the method at powerful pulsed spallation neutron source instruments providing intrinsic wavelength resolution.

\section{Measurements}

Wavelength-dispersive measurements of a number of reference samples with well known scattering characteristics have been performed at ICON at PSI, Switzerland (Kaestner et al., 2011). The instrument features an option in which wavelength resolution is achieved by means of a mechanical velocity selector with a wavelength resolution of about $\Delta \lambda / \lambda=15 \%$. The wavelength resolution is sufficient for grating interferometer dark-field measurements and provides good flux for imaging experiments. A wavelength can be selected from the cold incident spectrum starting from $\lambda_{\text {min }}=2.8 \AA$ with significantly decreasing intensities for longer wavelengths, limiting the useful range to a maximum wavelength $\lambda_{\max }$ of about $6 \AA$. ICON is also provided with a dedicated grating setup optimized for 4.1 A neutrons. The corresponding key parameters and components are a source grating $G_{0}$ with a period $p_{0}=$ $1076 \mu \mathrm{m}$ installed close to the pinhole of the instrument and a phase grating $G_{1}$ with a period of $p_{1}=7.97 \mu \mathrm{m}$ placed at a distance of $L_{1}=5.23 \mathrm{~m}$ downstream of the source grating. The analyser grating $G_{2}$ like the source grating $G_{0}$ is an absorption grating, but with the modulation period $p=4 \mu \mathrm{m}$, because with such a setup the modulation $p=p_{1} / 2$ (Pfeiffer et al., 2006). Reference samples consisting of monodisperse colloidal polystyrene (PS) particles suspended in a mixture of $\mathrm{H}_{2} \mathrm{O}$ and $\mathrm{D}_{2} \mathrm{O}$ with different well defined diameters and similar weight concentrations were used. The mixture of $\mathrm{H}_{2} \mathrm{O}$ and $\mathrm{D}_{2} \mathrm{O}$ (59:41) was chosen in order to enable a close matching of the

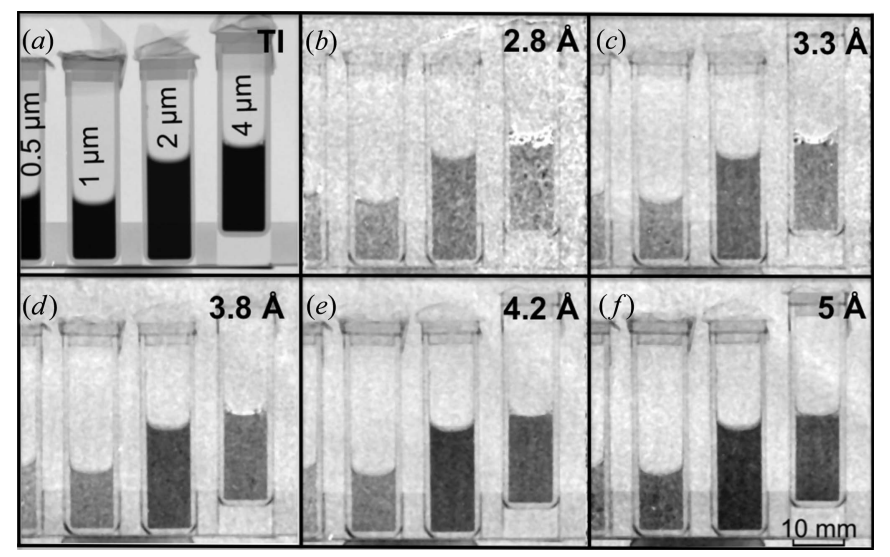

Figure 2

(a) Transmission image (TI) of the sample setup, indicating diluted particle sizes in the respective cuvettes, and corresponding dark-field images taken at wavelengths of (b) $2.8 \AA$, (c) $3.3 \AA$, (d) $3.8 \AA$, (e) $4.2 \AA$ and $(f) 5 \AA$. densities of the particles, with the aim of preventing particle sedimentation during the measurements. The original provided concentrations of the particles (NIST traceable standards) were increased to around $10 \%$ in order to achieve high contrast. PS particles with diameters of $0.5,1,2$ and $4 \mu \mathrm{m}$ were used. The samples were contained and sealed in quartz cuvettes, defining the sample thickness $t=5 \mathrm{~mm}$, and all samples could, because of the imaging nature and spatial resolution of the measurements, be exposed simultaneously. The field of view in the imaging setup was about $6.4 \times 6.4 \mathrm{~cm}$ wide, enough to expose four cuvettes with a width of about $1 \mathrm{~cm}$ at once (Fig. 2). The spatial image resolution provided by the imaging geometry and detector were of the order of $100 \mu \mathrm{m}$.

For five wavelengths from 2.8 to $5 \AA$, modulation scans were performed by a phase stepping procedure with 24 equidistant steps over a full period. The empty beam visibility decreases for wavelengths different from the design wavelength of $4.1 \AA$ but is well above $15 \%$ and is still sufficient for measurements everywhere in this range. Every scan, i.e. the exposure time required to achieve a single dark-field image providing the spatially resolved visibility information, required between 90 and $600 \mathrm{~s}$, depending on the wavelength, owing to the flux decrease at longer wavelengths (Kaestner et al., 2011). Measurements were repeated at three different sample-todetector (analyser grating) distances $L_{\mathrm{s}}$ of 30,360 and $720 \mathrm{~mm}$ with and without samples in place. Replacing $L_{\mathrm{s}}$ with $L_{\mathrm{s}}^{\prime}$ values of $2.00,1.86$ and $1.72 \mathrm{~cm}$ for wavelengths between 2.8 and $5 \AA$ in equation (3), this results in a range of probed correlation lengths from about 1.2 to $2.5 \mu \mathrm{m}$. The recorded modulations have been fitted and analysed for every pixel of the images of a scan individually using the PSI data analysis procedures for grating interferometer imaging with phase stepping (Pfeiffer $e t$ al., 2006). This procedure was applied for every wavelength and every sample distance separately.

\section{Results and discussion}

Examples of dark-field images at different wavelengths are shown in Fig. 2. An area of interest of about $1 \mathrm{~cm}^{2}$ was chosen for each sample, over which the visibility values represented in the dark-field images were averaged. The values $V_{\mathrm{s}}$ were normalized by the corresponding visibility values $V_{0}$ of the open beam images without sample and associated with the respective dark-field lengths $\xi$. The wavelength dependence of the scattering cross section $\Sigma$ can be written as

$$
\Sigma_{\mathrm{s}}=(3 / 2) \varphi_{\mathrm{V}} \Delta \rho^{2} \lambda^{2} r
$$

for spherical particles, where $\varphi_{\mathrm{V}}$ is the volume fraction, $\Delta \rho$ is the scattering length density contrast between the particles and the solvent, and $r$ is the particle radius. This wavelength dependence necessitates a normalization of $\ln \left(V_{\mathrm{s}} / V_{0}\right)$ with $\lambda^{2}$, in order to combine the data sets acquired at different distances $L_{\mathrm{s}}$. Values with matching dark-field lengths $\xi$ from different distance measurements were averaged. The resulting curves were fitted with theoretical curves according to 


$$
\begin{aligned}
\ln \left(V_{\mathrm{s}} / V_{0}\right) / \lambda^{2} & =\left(\Sigma_{\mathrm{s}} / \lambda^{2}\right)[G(\xi)-1] t \\
& =(3 / 2) \varphi_{\mathrm{V}} \Delta \rho^{2} r[G(r, \xi)-1] t
\end{aligned}
$$

The scattering length density contrast $\Delta \rho$ was calculated using data from the NIST database (http://www.ncnr.nist.gov/ resources/n-lengths/) to be $81.7 \mu^{-2}$ for the PS particles in the $\mathrm{H}_{2} \mathrm{O} / \mathrm{D}_{2} \mathrm{O}$ mixture used, and only the particle radius $r$ and the volume fraction $\varphi_{\mathrm{V}}$ were free fitting parameters.

The results of this procedure are presented in Figs. 3(a)$3(d)$ for particle sizes $d$ of 1,2 and $4 \mu \mathrm{m}$. In the case of particles with a diameter of $1 \mu \mathrm{m}$ (Fig. $3 a$ ) it can be seen that the size range that can be probed because of the amenable dark-field lengths $\xi$ in the experiments is beyond the maximum correlation length $2 r$ of the particles and hence $G(\xi)=0$. This leads to a constant value of $\ln \left(V_{\mathrm{s}} / V_{0}\right) / \lambda^{2}=-\left(\Sigma_{\mathrm{s}} / \lambda^{2}\right)=$ $-(3 / 2) \varphi_{\mathrm{V}} \Delta \rho^{2} r t$, which hence does not allow us to extract the particle size, shape or volume fraction. Assuming, however, the a priori known particle size $r=0.5 \mu \mathrm{m}$, the volume fraction could be extracted as $\varphi_{\mathrm{V}}=0.12$.

For the particles with diameters of $4 \mu \mathrm{m}$ (Fig. $3 b$ and $3 c$ ), on the other hand, the maximum particle correlation length could not be reached and the dark-field length range stays below the value of $4 \mu \mathrm{m}$. Also in this case a fit is not possible without $a$ priori knowledge additional to the scattering length density contrast. However, with the known particle size again a fit is possible, providing the volume fraction. Note, however, that the data points shown in Fig. 3(b) represent a running average of the actual extracted data points from the measurement. This means that points falling within the same correlation length according to the bin width of the wavelength scan were averaged, and, because the resulting data set appeared noisy, a smoothing procedure over every three consecutive data points was applied. Fitting the resulting curve provides a time-averaged concentration of the particles of $4 \%$. The actual data points, however, describe two different curves (Fig. $3 c$ ). One set of points can be related to the earlier measurement at $L_{\mathrm{s}}=$ $30 \mathrm{~mm}$, while the data points with higher values with respect to the correlation length are from the later performed measurements at longer sample-to-detector distances. Separate fits of the respective data points provide concentration values of first $5.2 \%$ and later only $3.3 \%$. For several experimental reasons the second and third distance scans were performed a significant time after the first run, and hence it can be concluded that the decreasing volume fraction measured was due to sedimentation taking place in the sample. This arises because the density of the liquid phase and the particles is not completely matched and the initial concentration of large particles was relatively high. The sedimentation in this sample, in contrast to the other samples, was clearly visible to the naked eye after the measurement. However, this result also underlines that inhomogeneities can be studied not only with spatial resolution but also simultaneously with a certain time resolution, allowing observation of processes on the corresponding length scales.

The diameter of the third set of particles represented was $2 \mu \mathrm{m}$, well within the probed range. The results are presented in Figs. $3(d)-3(f)$ with respect to the normalized visibility values $\ln \left(V_{\mathrm{s}} / V_{0}\right) / \lambda^{2}$, visibility values $V_{\mathrm{s}} / V_{0}$ and extracted correlation function $G$, respectively. Fig. $3(d)$ shows that in this case a fit without additional a priori values is possible, which
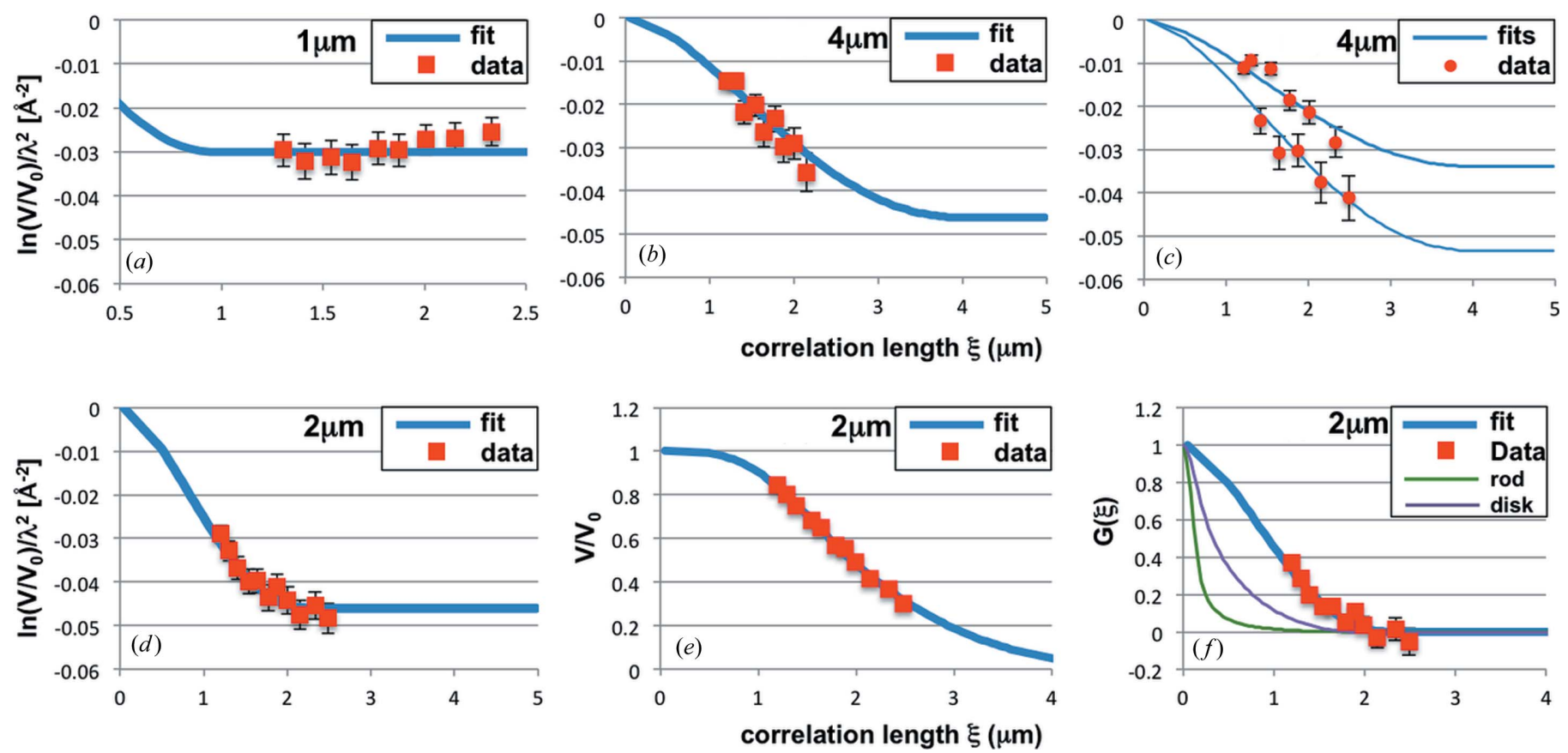

Figure 3

Resulting dark-field contrast curves depending on dark-field length $\xi$ with corresponding best fits for $(a) 1 \mu \mathrm{m}$ particles, $(b) 4 \mu \mathrm{m}$ particles with averaged signal, and (c) $4 \mu \mathrm{m}$ particles with independent fits for first distance and other distances, applied because of the obvious sedimentation effects of these large particles during the extended time for several scans. $(d)$ The respective data and fit for the $2 \mu \mathrm{m}$ particles, $(e)$ the corresponding relative visibility, and $(f)$ the real space correlation function $G$ corresponding to data points and the fit result as well as examples for other particle shapes of the same maximum correlation length. 
delivers values of $r=1.15 \mu \mathrm{m}$ and $\varphi_{\mathrm{V}}=0.08$. The particle size is hence within a $15 \%$ uncertainty, while the error for the volume fraction is harder to judge. The initial value of the concentration is $10 \pm 1 \%$ and is hence associated with an uncertainty of about $10 \%$. Additionally, some sedimentation cannot be excluded for this sample as well. However, the uncertainties in the current results compare favourably with reported corresponding values presented in a study comparing the accuracy of ultra-small-angle neutron scattering measured with doublecrystal diffractometers and SESANS (Rehm et al., 2013). Fig. 3(e) demonstrates how the measurement and fit compare on the measured scale of the relative visibility. It becomes obvious how much more sensitive the logarithmic evaluation (Fig. $3 d$ ) is with respect to signal deviations and error ranges. Fig. 3(f), however, underlines the power of the use of the correlation function in terms of the characterization of the particle shape. For the fits presented in Fig. 3, spherical symmetry was assumed for the particles described by the correlation function. Fig. 3(f) also shows the corresponding theoretical curves for correlation functions of randomly oriented disc- and rod-like particles, with the same maximum dimension and hence correlation length. This figure emphasizes that such structural features, referred to as the form factor in scattering, can be characterized well by the measurements.

\section{Conclusion}

It has been demonstrated experimentally with monodisperse micrometre sized particles that wavelength-dispersive darkfield neutron imaging with grating interferometers in principle enables full and quantitative characterization of structures beyond the direct spatial image resolution through small-angle scattering. This means that shape and size can be extracted in terms of the specific real space correlation function $G$ of the structure, and the total scattering power measured contains information about the scattering length density contrast and volume fraction of the scatterers (Strobl, 2014). Full information is available for each pixel of the dark-field images, and hence the SAS information is provided with additional macroscopic real space image resolution. However, it is also shown that the range of correlation lengths and hence structure sizes that can be probed in this way is rather limited for the currently used grating interferometer setups with neutrons. Although the size range of structures over which the method is sensitive spans more than an order of magnitude, as has been investigated previously (Betz et al., 2015) and is also obvious from the presented data, full quantification without $a$ priori knowledge about the investigated structures is with the used setup limited to a narrow range of about $1.5-2 \mu \mathrm{m}$. This range can, however, be tuned through a number of parameters, such as, for example, the gratings and distances utilized. The optimization of these parameters in combination with scanning procedures will have to be explored in order to enable promising ranges to be probed for broad application, particularly at pulsed spallation neutron sources with intrinsic wavelength resolution. The results, on the other hand, also show that significant quantitative information about scattering structures can be achieved when they are beyond the narrow range of probed correlation lengths, if, as is very often the case in small-angle scattering studies, some prior knowledge is available.

\section{References}

Andersson, R., van Heijkamp, L. F., de Schepper, I. M. \& Bouwman, W. G. (2008). J. Appl. Cryst. 41, 868-885.

Betz, B., Harti, R. P., Strobl, M., Hovind, J., Kaestner, A., Lehmann, E., Van Swygenhoven, H. \& Grünzweig, C. (2015). Rev. Sci. Instrum. 86, 123704.

Feigin, L. \& Svergun, D. (1987). Structure Analysis by Small-Angle $X$-ray and Neutron Scattering. New York: Plenum Press.

Grünzweig, C., David, C., Bunk, O., Dierolf, M., Frei, G., Kühne, G., Schäfer, R., Pofahl, S., Rønnow, H. M. R. \& Pfeiffer, F. (2008). Appl. Phys. Lett. 93, 112504.

Hilger, A., Kardjilov, N., Kandemir, T., Manke, I., Banhart, J., Penumadu, D., Manescu, A. \& Strobl, M. (2010). J. Appl. Phys. 107, 036101.

Kaestner, A., Hartmann, S., Kühne, G., Frei, G., Grünzweig, C., Josic, L., Schmid, F. \& Lehmann, E. H. (2011). Nucl. Instrum. Methods Phys. Res. Sect. A, 659, 387-393.

Manke, I., Kardjilov, N., Schäfer, R., Hilger, A., Strobl, M., Dawson, M., Grünzweig, C., Behr, G., Hentschel, M., David, C., Kupsch, A., Lange, A. \& Banhart, J. (2010). Nat. Commun. 1, 125.

Pfeiffer, F., Grünzweig, C., Bunk, O., Frei, G., Lehmann, E. \& David, C. (2006). Phys. Rev. Lett. 96, 215505.

Rehm, C., Barker, J., Bouwman, W. G. \& Pynn, R. (2013). J. Appl. Cryst. 46, 354-364.

Reimann, T., Muhlbauer, S., Schulz, M., Kaestner, A. \& Gruenzweig, C. (2015). Nat. Commun. 6, 8813.

Rekveldt, M. T. (1996). Nucl. Instrum. Methods Phys. Res. Sect. B, 114, 366-370.

Strobl, M. (2014). Sci. Rep. 4, 7243.

Strobl, M., Grünzweig, C., Hilger, A., Manke, I., Kardjilov, N., David, C. \& Pfeiffer, F. (2008). Phys. Rev. Lett. 101, 123902.

Strobl, M., Manke, I., Kardjilov, N., Hilger, A., Dawson, M. \& Banhart, J. (2009). J. Phys. D Appl. Phys. 42, 243001.

Strobl, M., Treimer, W. \& Hilger, A. (2004). Appl. Phys. Lett. 85, 488.

Strobl, M., Treimer, W., Hilger, A. \& Feye-Treimer, U. (2004). Physica $B$, 350, 155-158. 